\section{La paz inicia en las aulas: Análisis del sistema educativo como pilar fundamental de la sociedad en el posconflicto'}

Project "The Peace" starts in the classrooms

Ricardo Andres Cano-Andade 2 Lavralentina Robles-Tono ${ }^{3}$

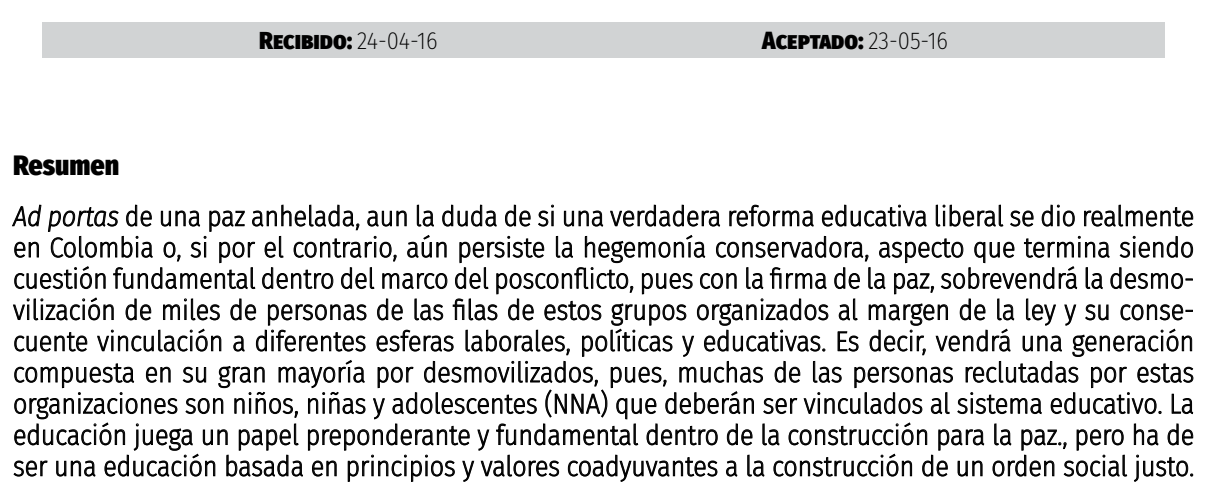
Palabras clave: diálogos de paz, educación, políticas públicas, sociedad, responsabilidad.

\section{Abstract}

Contributions from a longed-for peace, even the question of whether a true liberal education reform really

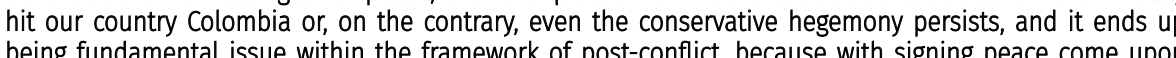
the demobilization of thousands of people from the ranks of these groups operating outside the law and its consequent attachment to different work areas, political and educational. That is, there will come a

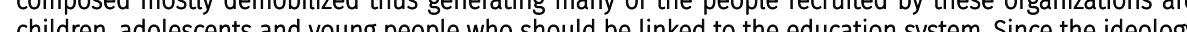
of the FARC keeps greater symmetry with liberal principles, distancing from conservative principles. Education plays an important and fundamental role in building peace in a society like Colombia where most but objective education, free of every aspect of fanaticism and ideological and religious dogmas

Keywords: peace talks, education, public policy, society, responsibility

Intorme de presentación del proyecto investigativo "La pazinicia en las aulas: analisis del sistema educativo como pilar fundamental de la

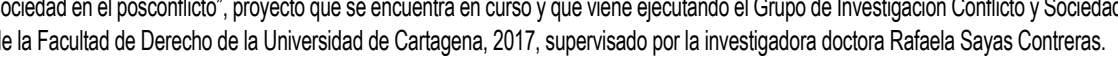

2 Estudiante de la Facultad de Dereccho de la Universidad de Cartagena. Senillerista de G Gupo de Invesigación Filosofia del Derecho.

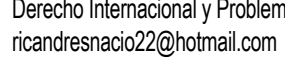

3 Estudiante de la Facultad de Derecho de la Universidad de Cartagena. Senillerista del Grupo de Investigación Conflicto y Sociedad de la Universidad de Cartagena.
valentina0514@hotmali.com

\section{PREGUNTA PROBLEMA}

¿Qué medida podrá tomar el Estado, teniendo en cuenta la cimentación de la paz y la inserción de las FARC de modo pleno a la vida civil; qué debe hacer frente a los dos modelos educativos existentes para que estos respondan a las necesidades de construir tejido social que haga posible que durante el posconflicto se erija una cultura de inclusión que integre a los sectores enfrentados a la par que expande el conocimiento?

\section{METODOLOGIA}

Se trata de una investigación cualitativa, descriptiva, de tipo analítico, documental, que se desarrollará a nivel dogmático teórico; la cual busca analizar bajo la inserción de una población desmovilizada y un nuevo ambiente de paz el modelo de educación adecuado para implementar después de firmada la paz. En el mismo marco teórico indagar a partir de la normatividad estudiada, los ajustes que se deberían implementar en materia de políticas públicas para fomentar la convivencia y garantizar una reintegración social de la población colombiana en el posconflicto.

\section{INTRODUCCIÓN}

Mientras se levantan aromas de paz y los acuerdos se vuelcan cada día como una posibilidad más latente y real, todas las direcciones se enfrascan en asumir las consecuencias económicas, políticas y jurídicas del Estado con los grupos implicados; todos los análisis van dirigidos en las medidas de reparación hacia las víctimas, las garantías de seguridad, la solución a los problemas de tierras y la solución de los distintos delitos cometidos. Todo lleva a imaginar una Colombia donde todos nos sujetamos de las manos con caras pintadas de patriotismo entonando canciones de paz, pero definitivamente no todo lo que parece, es.

El expresidente de la Comisión Europea Jacques Delors apunta que "la educación tiene la misión de capacitar a cada uno de nosotros sin excepciones en desarrollar todos sus talentos al máximo y a realizar su potencial creativo, incluyendo la responsabilidad de sus propias vidas y el cumplimiento de los objetivos personales" (1996) pero es precisamente ahi en donde radica uno de los principales problemas de nuestro país, pues resulta indis- 
pensable vivir en otro concepto de vida, al cual se arraiga Naciones Unidas y nos lleva a educar de otra forma y para otros fines.

Siendo así, es necesario enfocar el curso de esta investigación, en las distintas preguntas que se deben hacer en cuanto al modelo de educación después de firmada la paz, pues son las aulas en donde se comenzarán a dar los primeros contactos de familias de personas excombatientes, con familias víctimas, y resulta vital, estudiar los medios dentro del sistema para llevar la situación de la mejor manera. En base a lo anterior la pregunta problema en la que se basa este proyecto es ¿cuáles serían los ajustes que deben implementarse desde las políticas públicas educativas para mantener la sana convivencia y garantizar una reintegración social de la población colombiana en el posconflicto? Para tales efectos nos dedicaremos a analizar los siguientes tópicos, ¿es la educación núcleo fundamental para lograr la paz?, ¿qué políticas públicas podría asumir el Estado para lograr el fin de la paz?, ¿qué principios básicos debe asumir el sistema educativo? Y por último ¿puede el sistema educativo cambiar para la paz?

\section{EDUCACIÓN EN COLOMBIA}

Partimos del concepto de educación y su relación con la cultura, pues son dos conceptos que se presentan como indisolubles en el desarrollo del Estado colombiano, siendo imposible hablar de una cultura sin un concepto de educación. Para el Ministerio de Educación "La educación es un proceso de formación permanente, personal, cultural y social que se fundamenta en una concepción integral de la persona humana, de su dignidad, de sus derechos $y$ de sus deberes. Cultura es el conjunto de creencias, principios, normas y preceptos que predominan en un grupo de personas durante un tiempo determinado...". Toda cultura se articula a través de unos ejes centrales para dar respuestas a las preguntas esenciales del ser humano acerca de la divinidad, la concepción del hombre y de la sociedad, concepciones que perpetúan en las diferentes generaciones a través de la educación. Frente a esto, es necesario considerar que al utilizar la educación como medio para transmitir las concepciones culturales generacionales con el fin de que perduren en las nuevas y haya una continuidad histórica, por ello, todo modelo educativo es "intencional" y simultáneamente "tensional". Lo primero, porque parte de una concepción ideológica que indica que conceptos del hombre, la sociedad y la política maneja, lo segundo, porque refleja las distintas épocas de la evolución de cada sociedad y las crisis generadas por el choque de las concepciones de las diferentes generaciones en cada época histórica.

Lo anterior, quiere decir que los modelos de educación adoptados por cada Estado no son producto de parámetros escogidos por razones puramente éticas y altruistas, sino que, resultan de los intereses político-económicos predominantes. En esto la historia aporta grandes evidencias, pues, a través de los años se ha demostrado que el sistema educativo depende de la ideología que ostente el poder. Así, durante la época de la Hegemonía Conservadora de 1886 a 1930, la educación estaba orientada hacia un conocimiento controlado por la Iglesia Católica, quien tenía un gran poder político y social, pudiendo inclusive durante la vigencia del Concordato de 1887 escoger los libros de religión, los cuales debían tener la aprobación del obispo de la ciudad del colegio. Con la crisis del Partido Conservador y el ascenso al poder del Libera en los años 30 del siglo XX, se inician una serie de reformas con la intención de modernizar al país por medio de la secularización del Estado y la sociedad, a través de una educación laica. Es así cómo, en 1936, durante el gobierno de Alfonso López Pumarejo se reforma la educación, a través de la Reforma Constitucional de dicho año, que consagró las libertades de conciencia y de enseñanza y la Ley 32 del mismo año que le permitió al Estado la vigilancia y control de la educación. Esta reforma educativa liberal se complementó con la creación de dos establecimientos educativos públicos, la creación de la Escuela Normal Superior y la construcción de la Ciudad Universitaria, para el funcionamiento de la Universidad Nacional.

El artículo 68 de nuestra Constitución Política de 1991 consagra la doble naturaleza de la educación en Colombia: La enseñanza oficial o pública a cargo del Estado y el modelo de enseñanza particular o privado. Sin embargo, este artículo tiene un criterio de diferenciación en el inciso 4으, cuando establece que en los establecimientos del Estado ninguna persona podrá ser obligada a recibir educación religiosa, limitando esta prerrogativa a las instituciones públicas y dejando por fuera los establecimientos de educación superior de particulares. Esto significa que el principio del Estado laico introducido en la Carta del 91 no es absoluto, pues aplica solo para la educación pública, mientras que las instituciones privadas se rigen por el modelo de educación confesional. 
Actualmente hay 286 instituciones de educación superior registradas en el país, de las cuales 80 son públicas y 206 son privadas. Esto quiere decir que, de cara a un proceso de desmovilización y reinserción e integración a la sociedad civil y ante la escasez e insuficiencia de las instituciones oficiales, miles de excombatientes deberán ingresar a universidades privadas como alternativa para estudiar. Y aquí es donde se cuestiona si estos establecimientos educativos están preparados para un proceso de acogida institucional para los desmovilizados y si las bases de su modelo educativo son suficientes para afrontar las necesidades de esta nueva generación.

Muchas de las universidades privadas existentes en el país como el Colegio Mayor de Nuestra Señora del Rosario o la Universidad Javeriana, fueron fundadas a finales del siglo XVI y principios del siglo XVII por jesuitas franciscanos, quienes impartieron una educación de corte confesional que se conserva hasta el día de hoy. Esto explica por qué aún en muchas de las universidades privadas se incluyen dentro del pensum académico materias como "cultura franciscana", entre otras.

\section{EL PROBLEMA DE LA DOBLE NATURALEZA DE LA EDUCACIÓN EN COLOMBIA}

Desde la perspectiva de costos, el artículo 68 de la Carta del 91 contempla dos modelos: el de ENSEÑANZA OFICIAL -PÚBLICA-, donde el Estado es el propietario y el de ENSEÑANZA PARTICULAR -PRIVADO-. LOS dos modelos deben cumplir ciertos objetivos homogéneos y tener también unas reglas homogéneas; todo esto viene en la Ley 115/1994 (Ley de la Educación). Sin embargo, la propia Constitución tiene un criterio de diferenciación cuando dice que en los establecimientos del Estado ninguna persona podrá ser obligada a recibir educación religiosa, inciso $4^{\circ}$ del artículo 68 citado. Esto significa que el principio del Estado laico introducido en la Carta del 91 se desarrolla en la educación pública (como anteriormente se mencionó).

Con la introducción del cristianismo, la PAIDEIA griega se transforma en PAIDEIA cristiana y desde esa perspectiva solo era válido enseñar desde los dogmas de fe de los contenidos materiales de la educación; por lo tanto, se entendía que el modelo cristiano de educación era prepararnos mejor para merecer la salvación, conocimientos racionales de él; pero para poder crear una sociedad liberal, necesariamente el modelo religioso de base cristiana no sirve porque solo te prepara para pensar de determinada manera y solo de eso, porque por definición dogmática cualquier otra forma de pensamiento era hereje, esa es la gran limitante del modelo confesional de educación; asî pues, el gran problema de la historia de Colombia y América está en crear un modelo laico abierto a todos los debates del pensamiento sin marginar a nadie por cuestiones ideológicas o religiosas; establecer un modelo laico en Colombia fue un proceso largo y tortuoso. Con la Carta del 91 esto toma rango constitucional por lo que perfectamente se puede hablar tanto del ejercicio de la Libertad de Cátedra de cualquier temática como su debate. Pero no solo es el modelo laico; tenemos otra herencia cultural problemática: en el Concordato de 1887 se estableció la obligatoriedad de dar religión en todos los niveles educativos del país; por lo tanto, aceptar por un padre de familia matricular a su hijo en un colegio de moral confesional, implica aceptar que se va a ser educado bajo la religión del colegio donde va a ser matriculado.

\section{El artículo 69 de la Constitución Política especifica que:}

Autonomía universitaria. Investigación científica y acceso a la educación superior. Se garantiza la autonomía universitaria. Las universidades podrán darse sus directivas y regirse por sus propios Estatutos, de acuerdo con la ley. La ley establecerá un régimen especial para las universidades del Estado. El Estado fortalecerá la investigación científica en las universidades oficiales y privadas y ofrecerá las condiciones especiales para su desarrollo. El Estado facilitará mecanismos financieros que hagan posible el acceso de todas las personas aptas para la educación superior.

El anterior artículo es desarrollado por la Ley 30/1992. Por razones históricas, e modelo educativo colombiano nunca ha estado coordinado o ensamblado en sus distintos niveles; las universidades evolucionan por un lado y los colegios por otro; por ello, hay dos estatutos legales: la Ley 115/1994 para primaria y secundaria y la citada Ley 30 para las universidades; ello se evidencia en todas las universidades cuando los bachilleres que ingresan muestran carencias crecientes que se comprueban en las pruebas PISA, por ejemplo.

El otro aspecto importante es que las universidades se han visto envueltas en diálogos entre el modelo laico y el modelo confesional: la Iglesia no permitía perder el monopolio universitario tutelado en la Colonia, solo en la época en el que el Partido Liberal logró gobernar (1845) se crea el modelo totalmente laico copiado del modelo francés, con la creación de la Universidad Naciona en la época del gobierno de José Hilario López; por lo tanto, esa universidad 
fue vista como generadora de herejías y por ende de pecadores. Las universidades religiosas para luego volver a funcionar con la expedición de la carta de 1886. Con ocasión de la Guerra de los Mil Días las universidades públicas siguieron funcionando pero dejando de lado el laicismo. Las familias liberales optaron por crear universidades privadas laicas porque no querían que sus hijos recibieran educación confesional en establecimientos controlados por la Iglesia; en ese contexto nace el Externado de Colombia, después se crea la Universidad Libre, hasta que, poco a poco, durante la Revolución en Marcha, se recupera el sentido de lo laico en la Nacional, poniéndose a funcionar una educación pública orientada hacia lo laico, aunque no plenamente laica, por el Concordato de 1887 mencionado. Antes de la Carta de 91 las universidades públicas nacionales, departamentales o municipales eran institutos descentralizados, figura que llegó a su máximo desarrollo con el Decreto Ley 080/1980, expedido durante la presidencia de Julio César Turbay Ayala.

Otro aspecto a considerar es que el general Santander implantó el modelo universitario francés, allí se creó el MODELO NAPOLEÓNICO, porque es Napoleón quien lo inventa, lo sustenta y lo pone a funcionar. El Estado es dueño de todas las Universidades pero todos los docentes son empleados del Estado y ese aspecto es clave para entender por qué, un docente en Colombia es empleado público, así Napoleón quiso controlar a los profesores para que no lo criticasen. Junto a este existe el MODELO ALEMÁN basado en el denominado Seminario Investigativo alemán alrededor del cual se articula el proceso universitario y, el MODELO TUTORIAL inglés convertido en anglosajón. Tanto el modelo tutorial como el modelo alemán descansan en una absoluta libertad de cátedra, no en una idea preconcebida que hay que aprender a la fuerza, por medio del libre proceso de interacción docente (Tutor) dicente donde genera la búsqueda y construcción del saber. El modelo napoleónico exagera la clase magistral al convertirla en el eje de la práctica académica. Las universidades nacidas como entes autónomos, en el modelo napoleónico la pierden en virtud de que el Estado es el dueño de ellas y los docentes son empleados públicos, sometidos a un estatuto legal que rige la docencia.

\section{LA EDUCACIÓN EN COLOMBIA FRENTE A LA PA}

En uno de sus periódicos informes, la Unesco se refiere a la educación para la paz como el significado de educar "para la crítica y la responsabilidad, para la comprensión y el manejo positivo de los conflictos, asi como potenciar los valores del diálogo y el intercambio y revalorizar la práctica del cuidado y de la ternura, todo ello como una educación pro-social que ayude a superar las dinámicas destructivas y a enfrentarse a las injusticias...", así deja en claro el enfoque vital que debe tener la educación dentro de un proceso, como por ejemplo sería el de Justicia Transicional, pues después de acabado el conflicto es la educación el que posibilitará el cambio de una cultura de violencia a una cultura de aceptación.

El inconveniente más grande que presenta ahora mismo el proceso de paz es la inclusión de nuevas y escasas políticas educativas dentro de los diálogos y acuerdos. Una de estas, se evidenció en el año 2014, a través de la Ley 1732, por medio de la cual se estableció la CÁTEDRA DE LA PAZ, en todas las instituciones educativas de preescolar, básica y media como una asignatura independiente; no obstante en concordancia con el principio de autonomía universitaria cada institución de educación superior desarrollará tal cátedra de acuerdo a sus programas.

Ahora bien, de acuerdo al Decreto 1038 de 2015, por el cual se reglamenta la Cátedra de la paz, esta tendrá por objetivo "fomentar el proceso de apropiación de conocimientos y competencias relacionados con el territorio, la cultura, el contexto económico y social y la memoria histórica, con el propósito de reconstruir el tejido social, promover la prosperidad general y garantizar la efectividad, los principios, derechos y deberes consagrados en la Constitución". Además encaminada a generar aprendizajes en los siguientes componentes:

a) Cultura de la paz: se entiende como el sentido y vivencia de los valores ciudadanos, los Derechos Humanos, el Derecho Internacional Humanitario la participación democrática, la prevención de la violencia y la resolución pacífica de los conflictos.

b) Educación para la paz: se entiende como la apropiación de conocimientos y competencias ciudadanas para la convivencia pacífica, la participación democrática, la construcción de equidad, el respeto por la pluralidad, los Derechos Humanos y el Derecho Internacional Humanitario.

c) Desarrollo sostenible: se entiende como aquel que conduce al crecimiento económico, la elevación de la calidad de vida y al bienestar social, sin agotar la base de recursos naturales renovables en que se sustenta, ni deteriorar el ambiente o el derecho de las generaciones futuras a utili- 
zarlo para la satisfacción de sus propias necesidades, de acuerdo con el artículo 3 de la Ley 99 de 1993.

No obstante a lo anterior, los diálogos han tomado mayor inclinación hacia conversaciones que tienen como fin la búsqueda de prontas soluciones, en cuanto a problemáticas pasadas respecto a delitos, victimas, y demás, dejando por fuera políticas de no repetición como la culturización mediante a educación, claro está, no se pretende decir que los demás temas no son importantes, pero sí afirmar que políticas educativas de afianciamiento de una cultura de paz, resultarían beneficiosas tanto para la inclusión social de los grupos desmovilizados y sus familias como para el Estado para que las nueva generaciones no afronten el flagelo de la guerra, como la Declaración Universal de la ONU.

A esto se refiere en su columna del periódico El Tiempo Francisco Cajiao cuando afirma:

La paz social no se consigue con unos acuerdos de terminación de la guerra. Eso lo sabemos todos. Pero tampoco basta con hacer restitución de tierras, reparación de víctimas o desminado de los campos. Lo fundamental es educar a los niños y reeducar a quienes fueron entrenados para la muerte. Es en el alma, en el interior profundo de cada ser humano, donde residen las causas que tienen que superarse. Solo con procesos educativos será posible desterrar gradualmente el odio, la rabia, la desconfianza entre vecinos, la incapacidad de reconocer dignidad en el contradictor.

Expone Cajiao que la educación no ha pasado por La Habana, y es que no se ha traído a consideración tema alguno sobre la incorporación de los hijos de desmovilizados, familiares o incluso desmovilizados analfabetas a que entren en el sistema educativo, dar una mirada a la más reciente ley dada en el Estado referente al tema es retroceder al Decreto 128 de 2003 en su artículo 15 dejando de forma aún más preocupante las modificaciones al sistema de reincorporación hechas por el Decreto 0001391 de 2011 expedido por el actual presidente Juan Manuel Santos donde se olvida completamente de las políticas de educación y lo cual deja por lógica jurídica al primer decreto mencionado como la norma de aplicación a los grupos de familias desmovilizados después de firmados los acuerdos. La educación es la piedra fundante para la construcción de ese cese de violencia tan esperado; por ello, este proyecto propone tres líneas básicas que ponen a la educación como fundamento real de la nueva cultura del posconflicto. Estas son:

1. La educación como derecho de los sujetos reincorporados.

2. La educación como garantía de reincorporación a la sociedad

3. La educación como reparación y medida de no repetición del conflicto.

\section{LA EDUCACIÓN COMO DERECHO DE LOS DESMOVILIZADOS}

Aterrizar el tema nos lleva a trazar la ruta sobre el derecho de la educación de toda esa "comunidad" desmovilizada para la paz, y es que no se puede pretender llegar a tales fines sin entender que se debe realizar una labor social para garantizar la completa adaptación de dichas familias en la población civil y, no solo de ellos sino de la misma población civil con ellos.

Y es que nadie se ha puesto a pensar que después de firmados los tratados y la iniciación del posconflicto nos espera una revolución educativa. Jóvenes de la actualidad tendrán hijos pequeños que podrán estar sentados en un aula de clases donde posiblemente a su diestra se encuentre el hijo de un desmovilizado, y a su siniestra el hijo de una víctima afectada por ese desmovilizado; padres de la actualidad, es muy posible que sus hijos en un futuro compartan ideas y elaboren intensos debates en aulas universitarias con chicos desmovilizados que a toda costa defenderán los ideales que los hicieron levantar en armas y que es su bandera para alcanzar puestos políticos.

Sin embargo resulta llamativo considerar las garantías a este Derecho Fundamental que tiene cada persona en el territorio colombiano, y es que en cuanto a medidas de educación básica y segundaria el Estado, a pesar de ser mínimas, presenta soluciones respecto al tema, como se pueden notar en el artículo 15 del Decreto 128 de 2003 o el artículo 68 de la Ley 115 de 1994 donde se garantiza educación a los grupos de familia e incluso a los mismos desmovilizados que la requieran y se entenderá la educación como forma de rehabilitación social. Sin embargo, respecto a la educación superior las cosas cambian y es que desde el punto de vista constitucional la educación universitaria no es obligatoria, lo cual quita cualidades en cuanto el Derecho en sí, incluso despojándolo en ciertas situaciones de su carácter de Derecho Fundamental, lo cual solo queda a disposición de la autonomía brindada por el artículo 69 de la Carta a cada entidad de regirse de forma autónoma por sus propios estatutos y solo ceñirse de manera general a la Ley 30 de 1992. 
Con base a lo expuesto anteriormente es necesario adentrarse al manejo del Estado colombiano respecto a la educación como Derecho Constitucional, y este se encuentra regulado por el artículo 68 de la Constitución y la Ley 115 de 1994, y en cuanto a materia de posibilidades educativas de estudios básicos y secundaria el Estado ha desarrollado ciertas políticas, que en nuestra consideración no son suficientes, pero sí existen bases respecto al compromiso público con la educación escolar de los colombianos. Al momento de desarrollar y unir todos los preceptos anteriores con los grupos desmovilizados, es el Decreto 128 de 2003 en su artículo 15 el cual asegura a toda esta población estudios pero solamente de básica secundaria partiendo de lo dicho anteriormente que los estudios superiores no son obligatorios en Colombia.

Aquí se encuentra un conflicto que se necesita analizar, y es que no se puede pretender solamente garantizar la incorporación de estos grupos armados si no se garantiza de forma correcta y eficiente su Derecho de EDUCACIÓN. De esto, nace la segunda hipótesis:

La posible expedición de una ley que establezca cupos adicionales especiales en colegios y universidades públicas del país donde se garantice por lo menos el ingreso a dos estudiantes de comunidades desmovilizadas en los programas ofrecidos por cada entidad y cupos para todos en los colegios oficiales. También deben establecerse tales cupos en los establecimientos educativos del sistema de subsidio familiar. (CAFAM)

En datos recolectados por la Agencia Colombiana para la Reintegración (ACR) se obtienen resultados importantes sobre las tendencias educacionales de los grupos desmovilizados:

Desde 2003 se han desmovilizado alrededor de 57 mil personas de los diferentes grupos armados ilegales, incluyendo los paramilitares y las guerrillas, de los cuales el $84 \%$ ingresaron al proceso de reintegración del Gobierno. De estos, el $87 \%$ son hombres, $70 \%$ se encuentran entre los 26 y los 40 años y $72 \%$ no completaron el bachillerato (ACR, 2015). Se estima además que la tasa de desempleo de los desmovilizados es del $15 \%$ (ACR, 2015). Es importante tener en cuenta además que aproximadamente el $49 \%$ de los desmovi- lizados tienen hijos y que por lo tanto, el proceso de reinserción se debe realizar también con sus familias.

En esta perspectiva el seguimiento del Estado se ha desarrollado inadecuadamente ya que un alto porcentaje de desmovilizados desaparece de los programas, veamos:

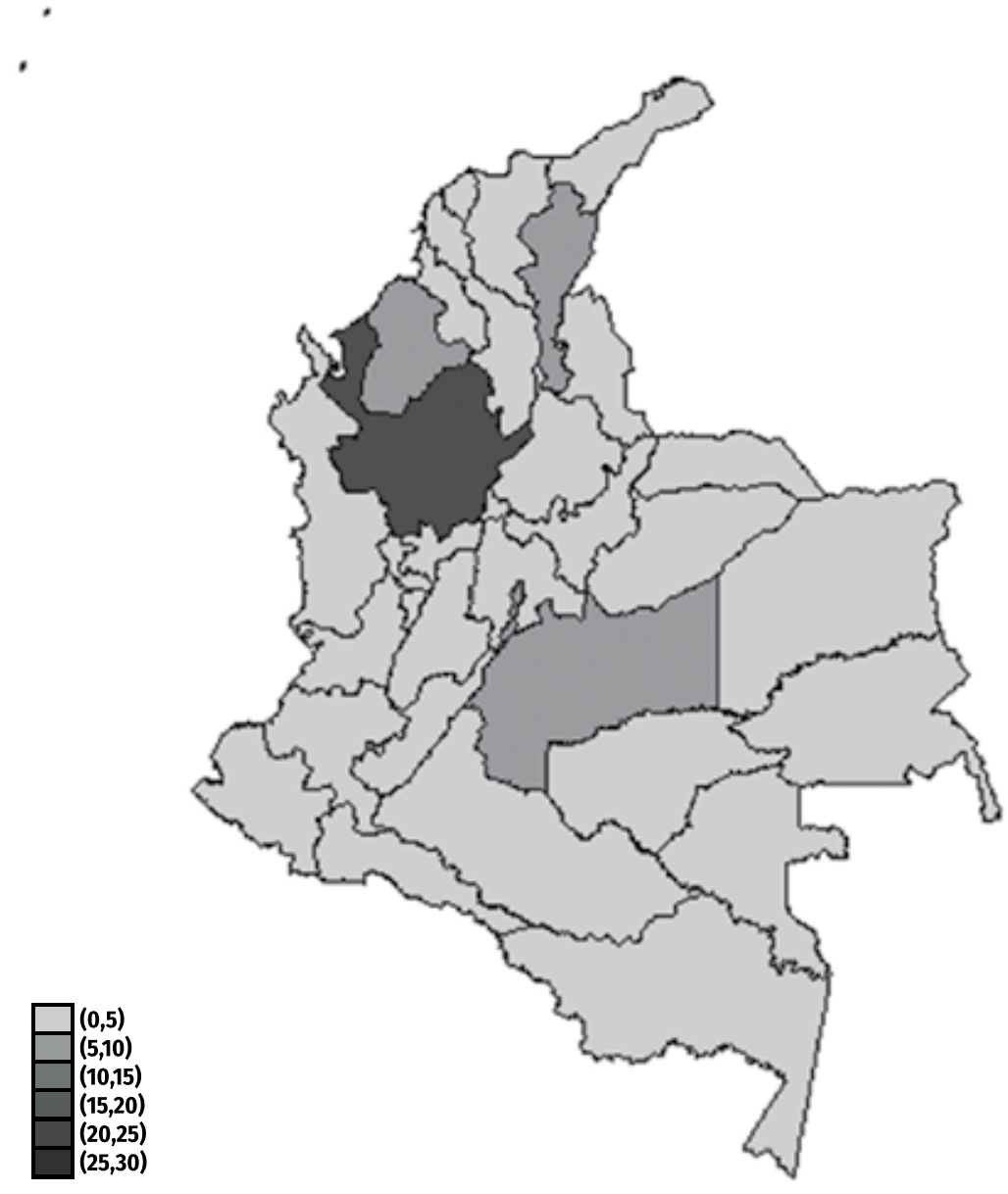

reintegración- Colombia, 2015

De acuerdo con el mapa anterior, quienes se desmovilizaron entre 2003 y junio de 2015, residen principalmente en Antioquia (20,4\%), Bogotá (10\%), Córdoba (6 \%) y Meta (5,3 \%). De acuerdo con el Centro de Memoria Histórica, existe concentración de desmovilizados principalmente en zonas urbanas. En Bolivar, por ejemplo, el $56,6 \%$ de los desmovilizados se encuentra en Cartagena y en 
el departamento del Atlántico, el 64 \% se concentra en Barranquilla mientras que en el departamento de Santander, el $76 \%$ se concentra en Bucaramanga.

Además, presentamos las siguientes figuras de la ACR que cargan datos de participación y niveles educativos de los desmovilizados con el programa.

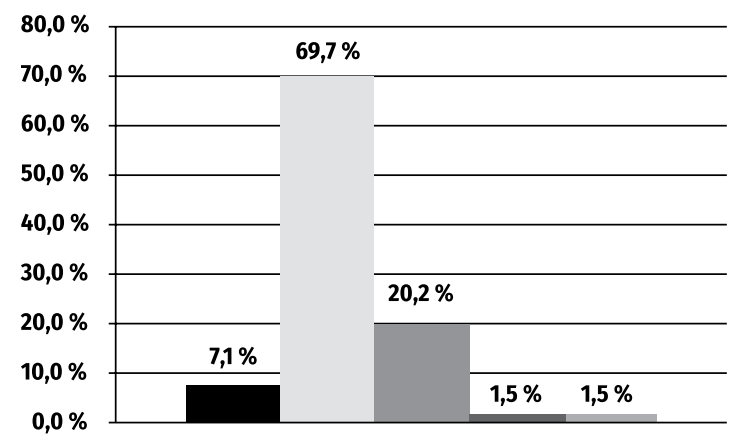

\section{$\begin{array}{ll}\text { Entre } 18 \text { y } 25 \text { años } & \text { Entre } 26 \text { y } 40 \text { años } \\ \text { E-Entre } 4 \text { y } 60 \text { años } & \text { Mayor de } 60 \text { años }\end{array}$}

Figura 1

ruente: "Estadísticas de rutas de reintegracion para desmovilizados" -Agencia colombiana para la eintegración- Colombia, 2015

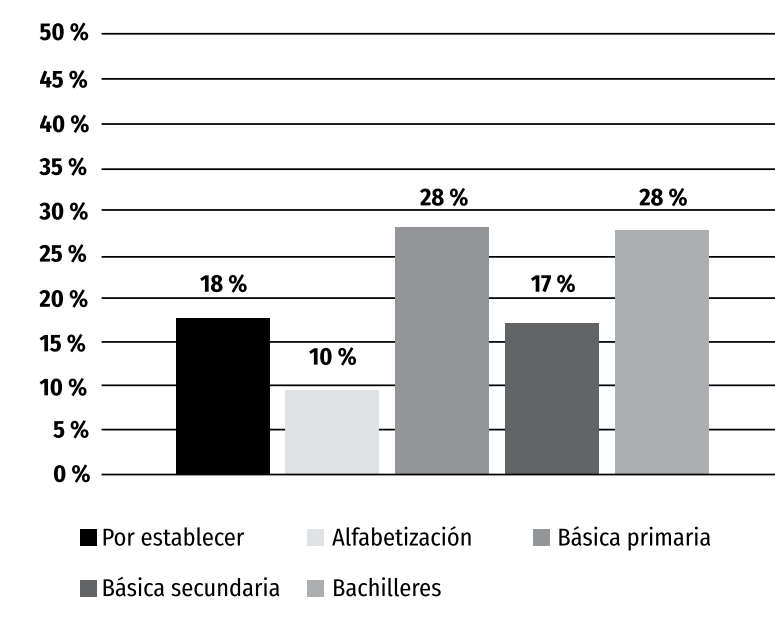

Figura

- Básica secundaria @ Bachilleres

Fuente: "Estadisticas de rutas de reintegración para desmovilizados" -Agencia colombiana para la reintegracion-Colombia, 2015

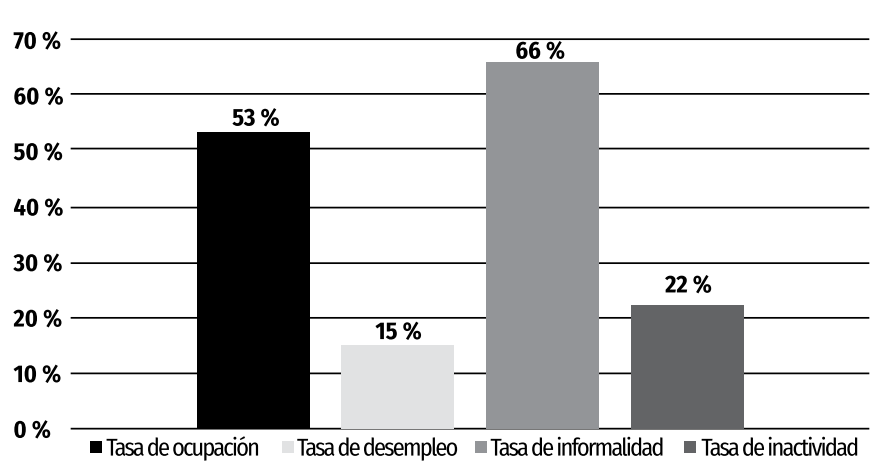

Figura 3

Fuente: "Estadísticas de rutas de reintegración para desmovilizados" -Agencia colombiana para la

En la Figura 1, se observa que la población desmovilizada que ingresó a la ruta de reintegración tiene una alta concentración de personas en edad productiva: $70 \%$ tienen entre 26 y 40 años. Aunque la Agencia Colombiana para la Reintegración (ACR) no presenta cifras acerca del número de menores de edad desmovilizados, el ICBF reporta que entre 1999 y 2014, 5.489 niños, niñas y adolescentes se desvincularon de grupos armados ilegales (ICBF, 2015).

La Figura 2 muestra que el $28 \%$ de los/as desmovilizados/as que ingresaron a la ruta de reintegración tienen educación media completa, es decir, bachillerato. Esto sugiere que menos de una tercera parte cumplía los requerimientos estándar para cursar un programa técnico y tecnológico de uno o dos años, en una entidad como el SENA. El $55 \%$ restante necesita procesos de formación más largos, iniciando el $38 \%$ desde primaria y $17 \%$ desde la educación media, que podría ser educación técnica laboral y de formación para el trabajo.

El bajo nivel educativo de esta población se refleja en sus oportunidades laborales. La Figura 3, muestra que la tasa de ocupación es del $53 \%$, cinco puntos porcentuales por debajo al promedio nacional (ACR, 2015). Por su parte la tasa de desempleo de los desmovilizados es del $15 \%$, seis puntos porcentuales por encima de la tasa nacional (9,1\%). La tasa de informalidad de esta población (66 \%) es mayor en 16 puntos porcentuales a la tasa naciona (49,5\%). Finalmente, se observa que los desmovilizados tienen una tasa de inactividad (que no trabajan, no estudian y no están buscando trabajo) del $22 \%$.

Con base en lo expuesto, es claro señalar que las formas de garantizar el Derecho de Educación de dichos grupos de familias desmovilizadas no ha sido el más eficiente, pero, se podría decir que las bases en cuanto a educación 
básica y secundaria tienen medios y formas que a opinión de nosotros con una adecuación a las políticas y mayor intensidad de vigilancia en relación a los programas del ICBF y de la ARC, se podría llegar a las metas trazadas del cumplimiento del Derecho.

Sin embargo, en cuanto a la educación superior las cosas son distintas, retomando la hipótesis planteada por el grupo, resulta inoperante la inclusión de cupos especiales en entidades de educación superior públicas mediante una ley, pues estas se encuentran facultadas de autonomía para regirse por sus propios reglamentos dados por el artículo 69 de la Constitución, y la Corte Constitucional aclara que:

"Finalmente, imponerles a las universidades -aunque sean públicas- una específica forma de selección de los aspirantes, y obligarlas a que adopten un sistema de cupos especiales, interfiere en el derecho de las universidades, consagrado en el artículo 69 de la Constitución, a "darse sus directivas y regirse por sus propios estatutos, de acuerdo con la ley". Porque de esa manera, se reduce el ámbito de aplicación de la garantía en tanto implica, en un sentido general, que las universidades ya no podrán reglamentar determinados asuntos de acuerdo con su propia concepción acerca de lo que es correcto, en el contexto de una formación educativa de nivel superior.... (Corte Constitucional, Sentencia T-110 de 2010, M.P. María Victoria Calle Correa)

Trayendo a colación las ideas planteadas anteriormente, el profesor Delors (1996) expone que "La educación para la paz será uno de los objetivos prioritarios de la escuela del futuro. La educación del siglo XXI, afirma la Comisión Internacional de la UNESCO, sobre la educación, debe apoyarse en cuatro pilares básicos..." tomando como referencia los estudios sobre educación de Delors, estos son:

Aprender a conocer: combinando una cultura general suficientemente amplia con la posibilidad de profundizar los conocimientos en un pequeño número de materias. Lo que supone, además, aprender a aprender para poder aprovechar las posibilidades que ofrece la educación a lo largo de la vida.

Aprender a hacer: a fin de adquirir no solo una calificación profesional sino, más generalmente, una competencia que capacite al individuo para hacer frente a gran número de situaciones y a trabajar en equipo. Pero, también, aprender a hacer en el marco de las distintas experiencias sociales o de trabajo que se ofrecen a los jóvenes y adolescentes, bien espontáneamente a causa del contexto social o nacional, bien formalmente gracias al desarrollo de la enseñanza por alternancia.

Aprender a vivir juntos: desarrollando la comprensión del otro y la percepción de las formas de interdependencia -realizar proyectos comunes y prepararse para tratar los conflictos-, respetando los valores del pluralismo, comprensión mutua y paz.

\section{- Aprender a ser para que florezca mejor la propia personalidad y se} esté en condiciones de obrar con creciente capacidad de autonomia: de juicio y de responsabilidad personal. Con tal fin, no menospreciar en la educación ninguna de las posibilidades de cada individuo: memoria, razonamiento, sentido estético, capacidades físicas, aptitudes para comunicar.

Se puede notar que el tercer punto trata sobre el aprender a vivir juntos, logrado solo a través de la educación, la inclusión al sistema educativo resultaría determinante en la resocialización de estos desmovilizados pues sería el contacto constante de reculturización donde se podrá aplicar terapia de convivencia tanto a civiles del común, víctimas afectadas y desmovilizados. Esa es la esencia de los artículos 68 y 69 de la Ley 115 de 1994, al referirse a la educación como medio de rehabilitación social.

\section{LA EDUCACIÓN COMO PRINCIPAL POLITICA DE NO REPETICIÓN DEL}

\section{CONFLICTO}

Este enunciado es el punto medular del análisis efectuado pues entre las políticas de no repetición que ofrecen los modelos de Justicia Transiciona convencional, la verdad histórica plasmada en programas sociales para desarrollar en el sistema educativo y libros con análisis histórico de los hechos para educar con el horizonte claro de recordar nuestro pasado, mantener los acuerdos presentes y mirar al futuro recordando el pacto de paz que se estaría firmando.

Desde la finalización de la Guerra Fría, la obtención de la paz duradera en situaciones posconflicto ha estado en la agenda de la Organización de las Naciones Unidas (ONU) y de muchos países, ya que el número de conflictos internos ha crecido. En la cumbre mundial celebrada en 2005, la ONU decidió 
crear una comisión para la construcción de la paz, la cual se inauguró oficialmente el 23 de junio de 2006. Esta comisión es la encargada de sugerir estrategias para lograr la paz y que esta se mantenga y no haya una recaída en los conflictos. Desde su creación, la ONU ha participado en varias misiones para lograr la paz en conflictos. El relativo éxito de algunas de las misiones (Bosnia y Herzegovina, Kosovo, El Salvador) contrasta con los esfuerzos fallidos en ciertos países (Sierra Leona y Ruanda), y de alli surge la pregunta por la efectividad de las estrategias para lograr la paz.

Pero curioso es observar políticas de paz utilizadas por dicha organización, donde sentar a un niño palestino con un israelí para que aprendan a convivir untos ha sido un choque demasiado fuerte a la culturización, muchos de los conflictos actuales terminan con la aceptación, y esta solo se logra mediante la convivencia diaria, y el ser educados juntos nos enseña a convivir.

Berdal y Malone citadas por Filipov (2006), dividen las causas del conflicto en dos categorías importantes: conflictos sociopolíticos y conflictos de identidad. La primera clasificación explica el conflicto según el punto de vista de la política y de la economía, afirmando que las razones sociopolíticas juegan un papel importante en el contexto de las causas para la reaparición del conflicto. Por el contrario, Collier sugiere que las principales causas del resurgimiento de un conflicto se basan en la identidad (Filipov, 2006, p.17)

El problema sociopolítico está ligado a la responsabilidad del Estado de garantizar la educación como Derecho fundamental que comprenda la política de resocialización para superar la crisis de identidad existente, pues como se identificaba al principio, el problema trasciende más allá de la devolución de tierras o el reparar en dinero a las víctimas, esto es crucial para la paz, pero el conflicto está presente por la crisis de identidad de la cultura colombiana ya que educar es devolver identidad a una cultura que no ha podido proporcionarla; Colombia, como tantas veces se ha dicho, ha sido una "Nación" a pesar de si misma.

\section{CONCLUSIONES}

Cargar al sector público de toda la responsabilidad en cuanto a la deficiencia educativa del país es incorrecto a sabiendas de la gran responsabilidad que carga el sector privado al mantener vivo en las aulas educativas el modelo confesional educativo y la represión al modelo liberal.

2. La creación de cupos especiales para familias desmovilizadas en las entidades públicas mediante una ley, es atentar contra su autonomía dada por la Constitución, pero sí mediante un acuerdo que puede ser dirigido por el Gobierno; las universidades en solidaridad al proceso pueden adherirse a un acuerdo de ceder cupos especiales en pro de asegurar la resocialización de todos estos grupos desmovilizados.

3. El plan de seguimiento a las familias desmovilizadas debe ser referenciado con un mayor cuidado a lo cual se podría proponer una entidad descentralizada en todo el país, la cual se encargue de todas la familias acentuadas en el sector de su vigilancia, y asi garantizar un estricto seguimiento a sus procesos sociales.

4. La educación debe ser vista como la principal política de no repetición de conflicto pues es dentro de las aulas donde la memoria se fortalece y la convivencia se ejercita.

\section{REFERENCIAS BIBLIOGRÁFICAS}

Agencia Colombiana para la Reintegración (2015). Estadísticas de rutas de reintegración para desmovilizados. Colombia.

Cajiao, F. (2015). Columna "La Educación no ha pasado por La Habana", Colombia, Periodico El Tiempo - 24 de marzo.

Corte Constitucional (2010). Sentencia T-110/2010. Colombia.

Corte Constitucional (2013). Sentencia T-666/2013. Colombia.

Delors, J. (1996). Educación: hay un tesoro escondido dentro. París: UNESCO.

Filipov, F. (2006). Post-conflict peace building: Strategies and lessons from Bosnia and Herzegovina, El Salvador and Sierra Leone. Some thoughts from the rights to

Instituto Colombiano de Bienestar Familiar (2015). Estadisticas de niños desmovilizados. Colombia.

Ministerio de Educación Nacional (2015). Concepto de Educación. Colombia.

Rodríguez, M. (1994). "Educar para la paz y la racionalidad comunicativa", a Educando para la paz: Nuevas propuestas", Granada, Universidad de Granada.

UNESCO (1995). "La educación para la paz, los derechos humanos y la democracia Declaración de la 44a reunión de la Conferencia internacional de Educación ratificada por la Conferencia General de la UNESCO", noviembre de 1995. 